\title{
Forschendes Lernen im Langzeitpraktikum - Bedingungsfaktoren der Unterstützung von Lehramtsstudierenden
}

\author{
Timo Beckmann, Timo Ehmke \\ Leuphana Universität Lüneburg
}

Zusammenfassung: Forschendes Lernen ist ein hochschuldidaktisches Konzept, an das hohe Erwartungen in Bezug auf die Professionalisierung von angehenden Lehrkräften gestellt werden. Empirisch ist jedoch wenig bekannt, unter welchen Bedingungen es erfolgreich ist. Anhand einer Befragung von $N=252$ Studierenden wird der Zusammenhang zwischen theoretisch relevanten Bedingungsfaktoren (Unterstützung im Forschungsprozess, Ziel- und Nutzungstransparenz, Bezug zur Praktikumsschule) und Zielsetzungen Forschenden Lernens (Reflexionsfähigkeit, Forschungskompetenz, Interesse an Wissenschaft) untersucht. Analysen mit einem Strukturgleichungsmodell zeigen, dass alle drei Bedingungsfaktoren relevant, aber differenziell unterschiedlich prädiktiv und damit von hoher Bedeutsamkeit für erfolgreiches Forschendes Lernen sind. Sie können so zur Weiterentwicklung der Angebote zum Forschenden Lernen beitragen.

Schlüsselbegriffe: Forschendes Lernen, Lehrkräftebildung, Schulbezug

\section{Research-Based Learning During a Long-Term Internship - Conditional Factors for Supporting Teacher Students}

\begin{abstract}
Summary: Research-based learning is a concept of higher education pedagogy, for which high expectations are set with regard to the professionalisation of prospective teachers. Empirically, little is known about the conditions under which research-based learning embedded in a long-term internship is successful. On the basis of a survey with $N=252$ students, the relationship between theoretically relevant variables (support in the research process, transparent objectives, connection to the school) and objectives of research-based learning (reflective competence, research competence, scientific interest) is examined. Path analyses show that all three conditions are relevant for successful research-based learning. Therefore, these conditions should be considered in the development of learning opportunities for research-based learning.
\end{abstract}

Keywords: Research-based learning, teacher education, connection to the school

Prinzipien des Forschenden Lernens sind in einer Vielzahl von Studiengängen integriert. Zurückgehend auf das Humboldtsche Ideal der Einheit von Forschung und Lehre an der Universität wird die Integration Forschenden Lernens seit den 70er-Jahren immer wieder eingefordert (BAK, 1970) und hat sich in den letzten Jahren zu einem zentralen hochschuldidaktischen Konzept entwickelt (Fichten, 2017). An Forschendes Lernen werden dabei vielfältige, oft überhöhte Erwartungen gestellt, und der systematische
Nachweis, dass diese Erwartungen erfüllt werden, fehlt meist. Forschendes Lernen dient als Methode zur Vorbereitung auf herausfordernde, jeweilig neue, komplexe und unstrukturierte Probleme (Zimbardi \& Myatt, 2012). Hierzu bearbeiten Studierende im research-based training Fragestellungen selbstständig bzw. in Teams unter Nutzung wissenschaftlicher Methoden (Healey, 2005; Huet, 2017). Aus lerntheoretischer Sicht kann dabei angenommen werden, dass eine solche, an konstruktivistischen Prin- 
zipien orientierte, situierte, anhand konkreter und authentischer Probleme gestaltete Situation lernwirksamer ist als rein passiv-rezipierende Lernformen (Fichten, 2017; Huber, 2009). Dieser konstruktivistischen Orientierung folgend, findet Lernen im research-based training in einer sozialen Umgebung statt (Piaget, 1976), in der Studierende fragend und nach Antworten suchend mit anderen Akteurinnen und Akteuren zusammenarbeiten und sowohl Wissen über das Fach erwerben als auch lernen zu lernen (Huet, 2017). Die vielfältigen Erwartungen an Forschendes Lernen systematisieren Gess, Deicke und Wesels (2017) zu Kompetenzzielen für Studierende. Die Studierenden sollen sowohl (a) fach- und themenspezifische Ziele erreichen, (b) metakognitive Kompetenzen aufbauen, (c) kognitive Forschungskompetenz in Bezug auf rezeptive bzw. generierende Forschungskompetenz entwickeln und (d) eine forschende Haltung aufbauen. Im Folgenden werden diese Ziele für die Lehrkräftebildung adaptiert und Bedingungen der Zielerreichung herausgearbeitet.

\section{Ziele Forschenden Lernens in der Lehrkräftebildung}

Auch in der Lehrkräftebildung erfährt das hochschuldidaktische Konzept des Forschenden Lernens kontinuierliche Aufmerksamkeit und ist Teil der Empfehlungen zentraler Kommissionen zur Reform der Lehrkräftebildung (Terhart, 2000; Wissenschaftsrat, 2001), sodass sich mittlerweile auch eine Reihe von Praxishinweisen zur Etablierung Forschenden Lernens finden (z. B. Lehmann \& Mieg, 2018; Obolenski \& Meyer, 2006; Schüssler et al., 2017). Im Rahmen der Einführung von Langzeitpraktika wurde in den letzten Jahren zudem an einer Vielzahl von Standorten nicht nur die Praxisorientierung im Studium gestärkt, sondern auch vermehrt Elemente des Forschenden Lernens integriert (z. B. Nordrhein-Westfalen, Niedersachsen).

Die Etablierung Forschenden Lernens geht auch in der Lehrkräftebildung einher mit einer Unschärfe des Begriffes und unterschiedlichen
Ausdifferenzierungen (Systematisierungen siehe bei Feindt, 2006; Koch-Priewe \& Thiele, 2009; Zimbardi \& Myatt, 2012) sowie Verhältnisbestimmungen zwischen wissenschaftlichem Wissen und Professionswissen (Hofer, 2013).

Über alle Typen hinweg zeichnet sich gemäß Fichten (2017) Forschendes Lernen in der Lehrkräftebildung durch drei zentrale Merkmale aus: (a) Selbstständigkeit, (b) Theoriebezug und (c) Reflexion. Studierende arbeiten - in zentralen Phasen selbstständig - an authentischen Forschungsproblemen im Praxisfeld Schule, wobei ein theoretischer Bezug zum Problem hergestellt wird. Entsprechend der oben dargestellten angenommenen Eignung Forschenden Lernens zur Bearbeitung von komplexen und unstrukturierten Problemen wird davon ausgegangen, dass die Lehrkräftebildung besonders als Einsatzort geeignet ist, da pädagogisches Handeln nicht standardisierbar ist und es einer kritischen Reflexion der (eigenen) pädagogischen Tätigkeit bedarf (Helsper \& Kolbe, 2002; Schön, 1983). Ansätze des Forschenden Lernens sollen so dabei unterstützen, die Verbindung zwischen Theorie und Praxis (Korthagen, 2010) - als eine der zentralen Herausforderungen der Lehrkräftebildung - für Studierende herzustellen und somit professionalisierend wirken. Ein forschender $\mathrm{Ha}$ bitus (Fichten, 2017) bei Lehrkräften zeichnet sich sowohl dadurch aus, dass diese ihren eigenen Unterricht beforschen als auch nach dem Studium ein Interesse an Wissenschaft haben und Zusammenhänge zwischen wissenschaftlichen Ergebnissen und ihrer Unterrichtspraxis sehen. Für diese Arbeit werden Zielsetzungen Forschenden Lernens unter Nutzung und Ergänzung der Kompetenzziele nach Gess et al. (2017) wie folgt systematisiert. Erstens die Entwicklung von Reflexionsfähigkeit (Z1) als Teil einer forschenden Haltung, die sich vor allem durch die Einnahme einer reflexiven Distanz zur Praxis ausdrückt (Fichten \& Meyer, 2014). Eine solche Distanz ermöglicht das Hinterfragen beruflicher Praxis und die Verbindung dieser mit theoretischem oder empirischem Wissen. Zweitens der Aufbau von Forschungskompetenz, die sich nicht nur auf die Rezeption von Forschungsergebnis- 
sen bezieht, sondern vor allem auf die Generierung von Forschungsergebnissen durch die eigene Planung und Durchführung von Forschungsvorhaben (Z2; Wissenschaftsrat, 2006). Drittens das ebenfalls mit einer forschenden Haltung in Verbindung stehende und dafür notwendige Interesse an Wissenschaft (Z3) und die Überzeugung, dass wissenschaftliche Arbeit zur Bearbeitung von relevanten Problemen der Schulpraxis einen Beitrag leisten kann und sich somit ein Nutzen wissenschaftlichen Wissens für professionelles Lehrkräftehandeln ergeben kann (Weyland \& Wittmann, 2015).

\section{Bedingungsfaktoren Forschenden Lernens in der Lehrkräftebildung}

Obgleich Forschendes Lernen ein an einer Vielzahl von Standorten etabliertes hochschuldidaktisches Konzept darstellt und theoretische Annahmen eine gewisse Wirksamkeit erwarten lassen, ist die empirische Erkenntnislage zu Bedingungen, unter denen Forschendes Lernen in der Lehrkräftebildung, insbesondere im Zusammenhang mit Praxisphasen, wirksam ist, gering (Fichten, 2017; Hofer, 2013; Köker \& Störländer, 2017; Rothland \& Boecker, 2014). Daher sollte sich „Forschung zu schulischen Praxisphasen verstärkt auf diesen Ansatz hin ausrichten“ (Weyland \& Wittmann, 2017, S. 26). Die Evaluation des Praxissemesters in Nordrhein-Westfalen kommt zu dem Schluss, dass die Studierenden „dem forschenden Lernen, welches die Hochschulen im Rahmen von Studienprojekten begleiten, [...] weniger Relevanz [beimessen] " (Göbel, Ebert \& Stammen, 2016, S. 7).

Als zentrale Bedingung und Herausforderung nennt Fichten (2017) die Schaffung von Motivation und der notwendigen Akzeptanz bei den Studierenden. So fragen Streblow und Barsch (2017) in Bezug auf die Einführung von Studienprojekten zum Forschenden Lernen im Kontext der Praxisphase: „Erkennen [die Studierenden] eine Sinnhaftigkeit in der Ausübung forschender Tätigkeiten für ihren späteren Berufsalltag?““ (ebd. S. 75). Auch Homt und van Ophuysen (2018) zeigen, dass die Sinnhaftigkeit häufig nicht erkannt wird. Insofern wird an dieser Stelle die Schaffung von Ziel- und Nutzungstransparenz (B1) als Bedingung vorgeschlagen.

Weiterhin wird davon ausgegangen, dass Forschendes Lernen dann professionalisierend wirkt, „wenn es professionell, d.h. auf Basis methodologischer Regeln und unter der Beachtung wissenschaftlicher Gütekriterien, betrieben" wird (Fichten, 2017, S. 32). Studierende müssen hierbei unterstützt werden (B2), da sie nicht selbstverständlich über die notwendigen Kompetenzen verfügen.

Die Praxisrelevanz (Riewerts et al., 2018) und damit auch die Bezüge zwischen Forschendem Lernen, den Erfahrungen im Praktikum und den Bedürfnissen der Praktikumsschule werden von den Studierenden oft nicht gesehen. Vielmehr erscheint es Studierenden vermutlich eher als zusätzliche Aufgabe, die nicht mit den Tätigkeiten in der Schule verknüpft ist (Ohm \& Zörner, 2019). Dieser Bezug zur Praktikumsschule (Z3) und damit auch der Bezug zu den Erfahrungen im Praktikum werden als dritte Bedingung angesehen, da somit das Lernen situiert werden kann.

Weyland und Wittmann (2017) fassen zusammen, dass Forschendes Lernen „einer intentionalen Verständigung zum Forschenden Lernen zwischen allen beteiligten AkteurInnen, einer prozessorientierten Vorbereitung und Begleitung und der Feststellung eines erkennbaren Nutzens insbesondere für die Studierenden" (S. 26) bedarf. Die drei hier benannten Bedingungen orientieren sich daran.

\section{Realisierung Forschenden Lernens im Rahmen des GHR-Lehramtsstudiums in Niedersachsen}

In mehreren Bundesländern wurden in den letzten Jahren Langzeitpraktika eingeführt (Weyland \& Wittmann, 2015). An diese mit schulischer Praxis verknüpften Phasen werden dabei unterschiedliche Anforderungen gestellt, wobei die Entwicklung einer unterrichtlichen Handlungskompetenz, die Reflexion über fremden oder eigenen Unterricht und die Reflexion über die eigene Eignung als Lehrkraft regelmäßige 
Ziele dieser Einführungen darstellen. Insofern sind die Planung, die Durchführung und die Reflexion von Unterricht wesentlicher Teil der Praktikumskonzepte. Insbesondere zur vertieften, theoriegeleiteten Durchdringung und Reflexion fremder oder eigener Praxis im Kontext von Praktikumssituationen wurden in mehreren Bundesländern Studienelemente, mit dem Ziel, Forschendes Lernen anzuregen oder zu unterstützen, etabliert (Weyland, 2012).

In Niedersachsen wurde zum Wintersemester 2014/2015 die Lehrkräftebildung entsprechend reformiert. Das Langzeitpraktikum (Praxisblock) ist im zweiten Mastersemester der Studiengänge für die Lehrämter an Grundschulen bzw. Haupt- und Realschulen angesiedelt. Der 18-wöchige Praxisblock, welcher als fachdidaktisches Unterrichtspraktikum konzipiert ist, wird durch je ein fachspezifisches Seminar, durch fachdidaktische Lehrende der Universität und Lehrkräfte mit Erfahrungen in der zweiten Ausbildungsphase vorbereitet, begleitet und nachbereitet. Ein mit insgesamt 15 ECTS kreditiertes und bewertetes Projektband vom ersten bis zum dritten Mastersemester ist ergänzend insbesondere zur Förderung des Forschenden Lernens angelegt (Niedersächsisches Ministerium für Wissenschaft und Kultur, 2014). Konzeptionelle Überlegungen zur Ausgestaltung der niedersächsischen Rahmenvorgaben an den Standorten liefern in Bezug auf Forschendes Lernen Spies, Michaelis, Gerhein und Hinsch (2017) für Oldenburg und Büsing, Gehrs, Mochalski, Nakamura und Treichel (2017) für Osnabrück.

An der Universität Lüneburg können die Studierenden im Bereich des Projektbandes zwischen Seminarangeboten zum Forschenden Lernen des Professionalisierungsbereichs und der Unterrichtsfächer wählen. Jedes Seminar folgt dabei einem eigenen inhaltlichen Schwerpunkt, jedoch ist ein wesentlicher Teil der Angebote im Projektband auf das inhaltliche Feld Umgang mit Heterogenität ausgerichtet. Beispiele für Seminartitel sind: „Forschendes Lernen im inklusiven Naturwissenschaftsunterricht“, „Multiprofessionelle Teams an der Schule“, „Mehr-BildungsSprachigkeit in der Migrationsgesellschaft?" .
Die Studierenden entscheiden sich für ein Seminarangebot mit Beginn des Studiums im ersten Mastersemester und schließen es mit einer Abschlusskonferenz im dritten Semester ab. Die Modulbeschreibung sieht die Konzeption, Durchführung und Dokumentation eines eigenen Forschungsprojektes vor. Eine Verknüpfung mit der Praktikumsschule ist nicht zwingend vorgesehen. Durch die durchgängige Begleitung der Studierenden über einen Zeitraum von drei Semestern im Rahmen einer Seminarveranstaltung zum Forschenden Lernen besteht für Lehrende die grundsätzliche Möglichkeit, im Seminar sowohl die Notwendigkeit von Forschung im Lehrkräfteberuf deutlich zu machen und somit für eine Zieltransparenz zu sorgen, als auch die Studierenden im gesamten Forschungsprozess zu unterstützen. Forschungsmethodische Grundlagen werden im ersten Bachelorsemester durch ein fachübergreifendes Modul Forschungsmethoden und zusätzlich durch ein Modul Methoden der empirischen Bildungsforschung am Ende des Bachelorstudiums angebahnt.

\section{Forschungsfragen}

Anknüpfend an die theoretische Diskussion, den Forschungsstand und die Situation in Niedersachsen soll mit dieser Studie deskriptiv untersucht werden:

1. Inwieweit werden die Ziele des Forschenden Lernens in Hinblick auf (Z1) Reflexionsfähigkeit, (Z2) Forschungskompetenz und (Z3) Interesse an Wissenschaft bei Studierenden erreicht?

2. Wie werden die Bedingungen des Forschenden Lernens bezogen auf die (B1) Ziel- und Nutzungstransparenz, die (B2) Unterstützung im Forschungsprozess und den (B3) Bezug zur Praktikumsschule wahrgenommen?

Aus einer analytischen Perspektive soll geprüft werden:

3. Inwieweit kann das Erreichen der Zielsetzungen Forschenden Lernens $(Z 1-Z 3)$ durch theoretisch relevante Bedingungsfaktoren (B1-B3) erklärt werden? 


\section{Methode}

\section{Design und Stichprobe}

Es wurden $N=252$ Studierende der Masterstudiengänge Lehramt an Grundschulen bzw. Haupt- und Realschulen befragt. Die Studie erfolgte im Kontext einer thematisch umfassenderen Evaluation der Einführung der Masterstudiengänge. Die Basisfragebögen wurden durch eine Gruppe von Expertinnen und Experten des Niedersächsischen Verbunds zur Lehrerbildung erstellt, den Standorten bereitgestellt und dort jeweilig durch eigene Studienelemente ergänzt. Bei den hier eingesetzten Items, welche durch die Autoren anhand inhaltlicher Entscheidungen zu Skalen zusammengefasst wurden, handelt es sich um Eigenentwicklungen des Niedersächsischen Verbundes zur Lehrerbildung. Die Erhebung erfolgte papierbasiert im Rahmen von Lehrveranstaltungen, an denen alle Studierenden der beiden befragten Kohorten teilnehmen sollten. Den am Erhebungstermin fehlenden Studierenden wurde angeboten, die Fragebögen kurz nach dem Erhebungstermin auszufüllen. Von den insgesamt 155 Studierenden, die im Sommersemester 2015 die Praxisphase absolviert haben, liegen 102 Rückmeldungen vor $(65,8 \%)$. Von den 178 Studierenden, die im Sommersemester 2016 die Praxisphase absolviert haben, liegen 150 Rückmeldungen vor $(84,3 \%)$. Entsprechend der Zusammensetzung der Studierenden am Standort haben 211 Studierende $(83,7 \%)$ angegeben, weiblich zu sein, und 29 Studierende $(11,5 \%)$ männlich. Insgesamt 176 Studierende $(69,8 \%)$ gaben an, Lehramt an Grundschulen zu studieren, 68 (27\%) gaben an, Lehramt an Hauptund Realschulen zu studieren.

\section{Untersuchungsinstrumente}

In der Befragung der Studierenden kamen drei Skalen auf Ebene der Zielsetzungen Forschenden Ler- nens (Reflexionsfähigkeit, Forschungskompetenz, Interesse an Wissenschaft) und drei Skalen zu Bedingungen Forschenden Lernens (Ziel- und Nutzungstransparenz, Unterstützung im Forschungsprozess, Bezug zur Praktikumsschule) zum Einsatz. In Tabelle 1 sind die Angaben zur Itemzahl und zur Reliabilität für jede eingesetzte Skala und die Korrelationen zwischen den Skalen angegeben. Die Reliabilitäten der Skalen können als ausreichend bis sehr gut angesehen werden $(.66 \leq \omega \leq .93)$. Das Antwortformat aller Items war: trifft gar nicht $z u(0)$, trifft eher nicht zu (1), trifft eher zu (2), trifft völlig $z u$ (3). In Tabelle 2 sind zu jeder Skala zwei Beispielitems dargestellt.

\section{Zielsetzungen Forschenden Lernens}

Die Zieldimension der Reflexionsfähigkeit (Z1) als Teil einer forschenden Haltung umfasst die wahrgenommene Entwicklung der wissenschaftsbasierten Reflexionsfähigkeit in Bezug auf Lehren und Lernen, die kritische Verknüpfung von Theorien, Konzepten und Diskursen mit dem pädagogischen Handeln in der Schule sowie die Einnahme einer kritisch-reflexiven Grundhaltung für das Handeln in der Schulpraxis (Fichten, 2017; Hofer, 2013; Schüssler \& Schöning, 2017).

Unter Forschungskompetenz (Z2) wird die wahrgenommene Kompetenz bei der Planung und Durchführung von Forschungsvorhaben, verbunden mit der Auswertung und Interpretation von Daten, verstanden. Insofern wird hier ein Schwerpunkt auf den generierenden Aspekt von Forschungskompetenz gelegt und nicht auf den rezeptiven (Gess et al., 2017; Klewin \& Koch, 2017).

Das Interesse an Wissenschaft (Z3) als weiterer Teil einer forschenden Haltung wird insbesondere darüber operationalisiert, ob die Studierenden angeben,

Tab. 1: Skalen Studierendenerhebung

\begin{tabular}{|c|c|c|c|c|c|c|c|c|}
\hline \multirow{2}{*}{ Skala } & \multirow{2}{*}{$\omega$} & \multirow{2}{*}{ Items } & \multicolumn{6}{|c|}{ Korrelationen } \\
\hline & & & $\left(Z_{1}\right)$ & $(\mathrm{Z} 2)$ & $\left(Z_{3}\right)$ & (B1) & (B2) & (B3) \\
\hline (Z1) Reflexionsfähigkeit & .67 & 3 & 1 & & & & & \\
\hline (Z2) Forschungskompetenz & .77 & 2 & $.58 * *$ & 1 & & & & \\
\hline (Z3) Interesse an Wissenschaft & .66 & 3 & $.52^{* *}$ & $.51^{* *}$ & 1 & & & \\
\hline (B1) Ziel- und Nutzungstransparenz & .78 & 2 & $.48^{* *}$ & $.48^{* *}$ & $.35^{* *}$ & 1 & & \\
\hline (B2) Unterstützung im Forschungsprozess & .93 & 4 & $.51^{* *}$ & $.51^{* *}$ & $31^{* *}$ & $.62^{* *}$ & 1 & \\
\hline (B3) Bezug zur Praktikumsschule & .74 & 4 & $.41^{* *}$ & $.19^{* *}$ & $.21^{* *}$ & $.24^{\star *}$ & $.35^{* *}$ & 1 \\
\hline
\end{tabular}

Anmerkungen: $\omega=$ McDonald's Omega.

** $p<.01$. 
Tab. 2: Beispielitems Skalen Studierendenerhebung

\begin{tabular}{|c|c|c|}
\hline \multicolumn{3}{|c|}{ Skala } \\
\hline$\left(\mathrm{Z}_{1}\right)$ & Reflexionsfähigkeit & $\begin{array}{l}\text { - Eine kritisch-reflexive Grundhaltung für mein eigenes Handeln in der } \\
\text { Schulpraxis einzunehmen.* } \\
\text { - Meine wissenschaftsbasierten Reflexionsfähigkeiten in Bezug auf } \\
\text { Lehren und Lernen konnte ich im Projektband (weiter)entwickeln.* }\end{array}$ \\
\hline (Z2) & Forschungskompetenz & $\begin{array}{l}\text { - Ein Forschungsvorhaben zu planen und durchzuführen.* } \\
\text { - Erhobene Daten auszuwerten, zu interpretieren und zu reflektieren.* }\end{array}$ \\
\hline$\left(\mathrm{Z}_{3}\right)$ & $\begin{array}{l}\text { Interesse an Wissen- } \\
\text { schaft }\end{array}$ & $\begin{array}{l}\text { - Die Arbeit im Projektband hat mein Interesse an wissenschaftlicher } \\
\text { Arbeit erhöht. } \\
\text { - Ich sehe grundsätzlich Möglichkeiten für Schulen, von den Forschungs- } \\
\text { projekten des Projektbandes zu profitieren. }\end{array}$ \\
\hline (B1) & $\begin{array}{l}\text { Ziel- und Nutzungs- } \\
\text { transparenz }\end{array}$ & $\begin{array}{l}\text { - Seitens der Hochschullehrenden wurde mir das Konzept des Projekt- } \\
\text { bandes mit seinen Zielsetzungen im Bereich des „Forschenden Lernens“ } \\
\text { erläutert. } \\
\text { - Es wurde der Nutzen forschungsmethodischer Kompetenzen bei Lehr- } \\
\text { kräften thematisiert. }\end{array}$ \\
\hline (B2) & $\begin{array}{l}\text { Unterstützung im } \\
\text { Forschungsprozess }\end{array}$ & $\begin{array}{l}\text { - Ich hatte die Möglichkeit, Fragen zur Entwicklung einer geeigneten } \\
\text { Fragestellung für mein Forschungsprojekt zu klären. } \\
\text { - Ich hatte die Möglichkeit, die Gestaltung der passenden Erhebungs- } \\
\text { instrumente für meine Fragestellung zu klären. }\end{array}$ \\
\hline & $\begin{array}{l}\text { Bezug zur Praktikums- } \\
\text { schule }\end{array}$ & $\begin{array}{l}\text { - Die zentralen Fragen/Themen meines Forschungsprojektes waren direkt } \\
\text { mit meinen Erfahrungen im Praxisblock verknüpft. } \\
\text { - Mein Projekt hat Themen bzw. Forschungsfragen meiner Praktikums- } \\
\text { schule aufgegriffen. }\end{array}$ \\
\hline
\end{tabular}

Anmerkungen: * Einleitung der Items mit: „Durch das Projektband habe ich folgende Kompetenzen im Bereich wissenschaftsbasierter Reflexion von Lehren und Lernen (weiter-)entwickelt:“.

dass sich ihr Interesse an wissenschaftlicher Arbeit (auch als berufliche Tätigkeit) erhöht hat und sie grundsätzliche Möglichkeiten sehen, dass Schulen von Ergebnissen des Forschenden Lernens profitieren können (Gess et al., 2017; Klewin \& Koch, 2017).

\section{Bedingungsfaktoren Forschenden Lernens}

Ziel- und Nutzungstransparenz (B1) zur Verdeutlichung des Nutzens wissenschaftlichen Wissens für Lehrkräfte umfasst, ob die Ziele Forschenden Lernens erläutert wurden und ob darüber hinaus der Nutzen forschungsmethodischer Kompetenzen bei Lehrkräften thematisiert wurde. Es steht im Mittelpunkt, ob die Studierenden in den begleitenden Seminarveranstaltungen dazu angeregt wurden, ihr Projekt in einen allgemeineren Kontext zu setzen, und somit die Sinnhaftigkeit von Forschendem Lernen für die Studierenden deutlich werden kann. Gerade diese Sinnhaftigkeit wird von Studierenden häufig nicht gesehen (Streblow \& Barsch, 2017).
Unterstützung im Forschungsprozess (B2) dient der Steigerung der Professionalität der Forschung und setzt sich dabei aus mehreren forschungspraktischen Aspekten zusammen (Fichten, 2017). Es wird somit die Unterstützung im konzeptionellen und empirischen Teil des Forschungsprozesses (Entwicklung der Fragestellung, Auswahl von Methoden, Datenerhebung und Datenauswertung) abgebildet.

Bezugzur Praktikumsschule (B3) im Forschenden Lernen wird darüber modelliert, ob Themen der Praktikumsschule aufgegriffen werden, die Praktikumsschule Interesse am Projekt gezeigt hat und einen Einfluss auf Anlage und Umsetzung des Projektes hatte sowie ob zentrale Fragen des Projektes mit eigenen Erfahrungen verknüpft waren. Insofern steht hier die Praxisrelevanz (Riewerts et al., 2018) sowie Authentizität (Fichten \& Meyer, 2014) und damit die Schaffung inhaltlicher Anschlussfähigkeit zur Steigerung der Sinnhaftigkeit für die Studierenden im Vordergrund. Ein Einbezug der Praktikumsschule wird zudem als Indikator für einen Verständnisprozess zwischen den beteiligten Akteurinnen und Akteuren angesehen (Weyland \& Wittmann, 2017). 


\section{Verfahren zur Datenanalyse}

Zur Prüfung der Passung der Daten zur theoretisch angenommenen Struktur und zur Bearbeitung der Fragestellung 3 (Zusammenhänge zwischen Bedingungsfaktoren und Zieldimensionen) wurde ein latentes Strukturgleichungsmodell mit der Software Mplus 7.3 (Muthén \& Muthén, 2017) modelliert. Fehlende Werte wurden hierbei mittels FIML geschätzt. Die Modellgüte wird dabei anhand verschiedener Kriterien bewertet. Hierbei wird nicht alleine auf den relativ sensitiven $\chi^{2}$-Test eingegangen, sondern die Modellpassung wird auch anhand von robusteren Maßen wie dem Verhältnis des $\chi^{2}$-Wertes und den Freiheitsgraden ( $d f$; Schermelleh-Engel, Moosbrugger \& Müller, 2003) geprüft. Ergänzend wurde ein Modell unter Kontrolle der Kohorte gerechnet, wobei die erreichte Varianzaufklärung nicht bzw. marginal steigt. Daher wird dieser Effekt hier nicht weiter betrachtet. Deskriptive Analysen zu den Fragestellungen 1 und 2 wurden mit SPSS 25 durchgeführt.

\section{Ergebnisse}

Tabelle 3 fasst die deskriptiven Befunde für die ersten beiden Fragestellungen zusammen. Die Spalten 2-5 beinhalten die prozentualen Verteilungen der Skalenmittelwerte in den jeweiligen Intervallen. Die Zeilenprozente addieren sich jeweils zu $100 \%$.

\section{Forschungsfrage 1: Zielsetzungen Forschenden Lernens}

Aussagen zur Steigerung der Fähigkeit zur Reflexion und der Verbindung theoretischer Konzepte und praktischem Handeln in der Schule stimmen $54,3 \%$ der Studierenden (eher oder völlig) $\mathrm{zu}(M=1.50, S D=0.81 ; \mathrm{Z1})$. Bezogen auf eine Steigerung der eigenen Forschungskompetenz (Z2) fällt die Einschätzung der Studierenden ähnlich aus. So stimmen mehr als die Hälfte der Studierenden (56\%) entsprechenden Aussagen (eher oder völlig) zu $(M=1.73, S D=0.88)$. Einer Steigerung des Interesses an Wissenschaft (Z3) stimmen hingegen nur knapp ein Drittel $(31,5 \%)$ der Studierenden (eher oder völlig) zu $(M=1.24, S D=0.80$; Tab. 3).

\section{Forschungsfrage 2: \\ Bedingungsfaktoren Forschenden Lernens}

Die Ziele und der Nutzen (B1) wurden von weniger als 2/3 der Studierenden als (eher oder völlig) transparent wahrgenommen (61,9\%; $M=1.77, S D=0.87)$. Die Unterstützung im Forschungsprozess (B2) wird von den Studierenden überwiegend positiv bewertet. So stimmen 73,2\% der Studierenden eher oder völlig zu, dass sie während ihres Projektes unterstützt wurden $(M=2.04, S D=0.85)$. Einen Bezug zum Praktikum (B3) konnten allerdings weniger als ein Viertel $(19,7 \%)$ der Studierenden eher oder völlig herstellen $(M=0.99, S D=0.74$; Tab. 3).

Bislang wurde gezeigt, dass sich die Projekte in Bezug auf die durch die Studierenden wahrgenommenen Bedingungen unterscheiden und nur ein Teil der Studierenden von der Erreichung der Ziele Forschenden Lernens berichten.

Tab. 3: Deskriptive Ergebnisse

\begin{tabular}{|c|c|c|c|c|c|c|}
\hline & $\leq 0.75$ & $0.75 \leq 1.5$ & $1.5 \leq 2.25$ & $2.25 \leq 3$ & $M$ & $S D$ \\
\hline $\begin{array}{l}\text { Zielsetzungen Forschenden Lernens } \\
\text { (Z1) Reflexionsfähigkeit } \\
\text { (Z2) Forschungskompetenz } \\
\text { (Z3) Interesse an Wissenschaft }\end{array}$ & $\begin{array}{l}20,5 \% \\
13,7 \% \\
34,8 \%\end{array}$ & $\begin{array}{l}25,1 \% \\
30,3 \% \\
33,6 \%\end{array}$ & $\begin{array}{l}35,1 \% \\
31,5 \% \\
17,6 \%\end{array}$ & $\begin{array}{l}19,2 \% \\
24,5 \% \\
13,9 \%\end{array}$ & $\begin{array}{l}1.50 \\
1.73 \\
1.24\end{array}$ & $\begin{array}{l}0.81 \\
0.88 \\
0.80\end{array}$ \\
\hline $\begin{array}{l}\text { Bedingungsfaktoren Forschenden Lernens } \\
\text { (B1) Ziel- und Nutzungstransparenz } \\
\text { (B2) Unterstützung im Forschungsprozess } \\
\text { (B3) Bezug zur Praktikumsschule }\end{array}$ & $\begin{array}{r}10,9 \% \\
9,2 \% \\
45,6 \%\end{array}$ & $\begin{array}{l}27,2 \% \\
17,6 \% \\
34,7 \%\end{array}$ & $\begin{array}{l}36,8 \% \\
34,5 \% \\
15,5 \%\end{array}$ & $\begin{array}{r}25,1 \% \\
38,7 \% \\
4,2 \%\end{array}$ & $\begin{array}{l}1.77 \\
2.04 \\
0.99\end{array}$ & $\begin{array}{l}0.87 \\
0.85 \\
0.74\end{array}$ \\
\hline
\end{tabular}

Anmerkungen: Itemausprägungen: trifft gar nicht $z u$ (0), trifft eher nicht zu (1), trifft eher $z u$ (2), trifft völlig zu (3). 


\section{Forschungsfrage 3:}

Zusammenhänge zwischen den Bedingungsfaktoren und Zielerreichung Forschenden Lernens

Mit der dritten Fragestellung soll geprüft werden, inwieweit die drei Bedingungsfaktoren für das Erreichen der Zielsetzungen prädiktiv sind. Um diese Fragestellung zu prüfen, wurden in einem Strukturgleichungsmodell alle Faktoren gleichzeitig berücksichtigt und die spezifischen Vorhersagebeiträge berechnet (Abb. 1).

Das Modell geht sowohl von einer dreifaktoriellen Struktur der Bedingungsfaktoren als auch der Zielsetzungen Forschenden Lernens aus. Es lässt Pfade der drei Bedingungsfaktoren zu jeweils allen drei Zielsetzungen zu. Die fitWerte des Modells können insgesamt als ausreichend bis gut eingeschätzt werden $\left(\chi^{2}=239.18\right.$, $d f=120, p<.01, \chi^{2} / d f=1.99, \mathrm{CFI}=.95, \mathrm{TLI}=$
.94 , RMSEA $=.06$, SRMR $=.04)$. Der signifikante $\chi^{2}$-Test wird durch das robustere Maß des $\chi^{2} / d f$-Verhältnis ergänzt, der knapp zu niedrige TLI wird akzeptiert.

Durch die drei Bedingungsfaktoren lässt sich substanziell Varianz für jede der drei Zieldimensionen aufklären. Die Höhe der Koeffizienten des Strukturgleichungsmodells belegen, dass für die wahrgenommene Stärkung der reflexiven Distanz sowohl die Ziel- und Nutzungstransparenz $(\beta=.39)$ als auch der Bezug zur Praktikumsschule $(\beta=.40)$ jeweils einen spezifischen Vorhersagebeitrag leisten. Mit 54\% Varianzaufklärung kann ein hoher Anteil der Unterschiede in der Beantwortung erklärt werden. Die Studierenden berichten insofern umso häufiger von einer Steigerung der Fähigkeit, Theorien und Schulpraxis kritisch miteinander zu verknüpfen, je intensiver die bzw. der Lehrende die Ziele des Forschenden Lernens trans-

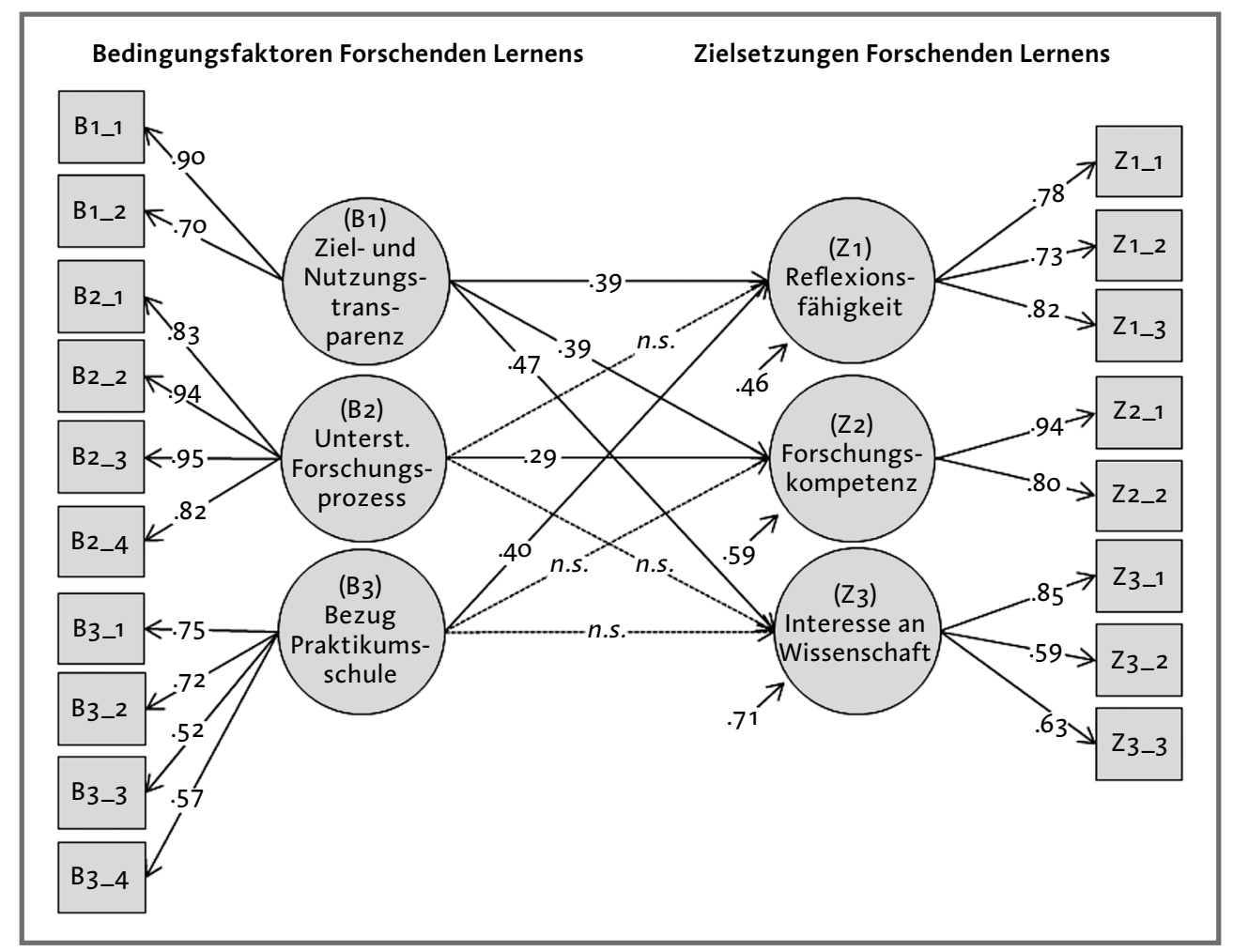

Abb. 1: Strukturgleichungsmodell zur Vorhersage der Skalen: Reflexionsfähigkeit, Interesse an Wissenschaft, Forschungskompetenz. 
parent gemacht hat und je enger das Forschungsprojekt mit dem Praktikum vernetzt ist. Für den wahrgenommenen Aufbau der Forschungskompetenz ist insbesondere die Zielund Nutzungstransparenz ( $\beta=.39)$ relevant, aber auch die Unterstützung im Forschungsprozess $(\beta=.29)$ verfügt über einen bedeutsamen Vorhersagebeitrag. Die Studierenden schätzen die selbst wahrgenommene Verbesserung ihrer Forschungskompetenz umso höher ein, je transparenter ihnen die Ziele des Forschenden Lernens gemacht wurden und je stärker sie von ihrer bzw. ihrem Lehrenden im Forschungsprozess unterstützt wurden. Die Varianzaufklärung beträgt hier $41 \%$. Die Varianz der Steigerung des Interesses an Wissenschaft kann mit 29\% am geringsten aufgeklärt werden. Prädiktiv ist hier allein die Ziel- und Nutzungstransparenz $(\beta=.47)$. Je stärker die Lehrenden die Ziele des Forschenden Lernens deutlich machen, desto stärker steigt auch das Interesse an Wissenschaft und der Wahrnehmung, dass Schulen durch Forschendes Lernen profitieren können.

Insofern zeigt sich, dass alle Bedingungen relevant für die Ergebnisse Forschenden Lernens sind. Die Pfade von Unterstützung im Forschungsprozess und Bezug zum Praktikum auf die weiteren Zieldimensionen leisten keinen signifikanten Aufklärungsbeitrag. Ein hoher Praktikumsbezug führt somit noch nicht zu einer Erhöhung der wahrgenommenen Forschungskompetenz und auch nicht zur Erhöhung des Interesses an Wissenschaft. Eine hohe instrumentelle Unterstützung hat nur in Bezug auf die Forschungskompetenz Erklärungskraft.

\section{Diskussion}

Zusammenfassend lässt sich festhalten, dass die befragten Studierenden auf der Ebene der theoretisch relevanten Bedingungsfaktoren des Forschenden Lernens, wie sie unter Bezug auf Gess et al. (2017) herausgearbeitet wurden, überwiegend von einer vorhandenen Unterstützung im Forschungsprozess berichten, sie erhalten beispielsweise konkrete Hilfe bei der Auswahl von
Methoden oder der Auswertung von Daten. Bei einem größeren Teil der Studierenden besteht aus ihrer Sicht nur ein geringer Bezug des Forschenden Lernens zur Praktikumsschule. Die Chancen, die sich durch die Gleichzeitigkeit der Praxisphase und des Forschenden Lernens für Schulen ergeben, werden insofern nur zum Teil genutzt. Auch werden Ziele und Nutzen Forschenden Lernens nur für einen Teil der Studierenden transparent, dies ist insofern erwartungsgemäß, da aus bisherigen Studien bekannt ist, dass Studierende die Sinnhaftigkeit des Forschenden Lernens nicht immer sehen (Homt \& van Ophuysen, 2018; Streblow \& Barsch, 2017). Auf der Ebene der Zielsetzungen des Forschenden Lernens gelingt bei etwa der Hälfte der Studierenden ein Aufbau der wahrgenommenen Forschungskompetenz. Gleichzeitig berichten die Studierenden seltener davon, dass durch die Arbeit im Projektband ihr Interesse an Wissenschaft erhöht wurde bzw. dass sie Möglichkeiten für Schulen sehen, vom Forschenden Lernen im Projektband zu profitieren. Auch in Bezug auf die Entwicklung der eigenen Reflexionsfähigkeit durch das Forschende Lernen im Projektband sind die Studierenden kritisch. Dies ist insofern relevant, da diese beiden Aspekte für forschende Lehrkräfte (Klewin \& Koch, 2017) von besonderer Bedeutsamkeit sind und beide Facetten mit Kompetenzzielen Forschenden Lernens in Verbindung stehen (Gess et al., 2017). Dieser Befund ist zudem ein Indiz dafür, dass Studierende im Praxissemester die Doppelrolle aus Involviertheit und Distanz nur schwer bewältigen können. Es kann vielmehr vermutet werden, dass bei vielen Studierenden im Langzeitpraktikum der selbst durchgeführte Unterricht wichtiger eingeschätzt wird als die Einnahme einer reflexiven, wissenschaftsbasierten Distanz (Rothland \& Boecker, 2014). Eventuell treffen Studierende während des Praktikums auf zu viele handlungsnahe Herausforderungen, sodass eine wissenschaftsbasierte und vertiefte Durchdringung spezifischer Fragen von Studierenden als wenig hilfreich wahrgenommen wird. Mithilfe eines Strukturgleichungsmodells konnte ge- 
zeigt werden, dass durch die drei theoretisch begründeten Bedingungsfaktoren zwischen $29 \%$ und $54 \%$ der Varianz der drei Zieldimensionen aufgeklärt werden kann. Der Aspekt der Ziel- und Nutzungstransparenz ist dabei für alle drei Zieldimensionen relevant und damit zentral in der Gestaltung Forschenden Lernens. Demnach kann nicht erwartet werden, dass Studierende selbstständig den Nutzen Forschenden Lernens für sich erkennen und somit motiviert sind (Fichten, 2017), sondern es wichtig ist, dass Lehrende diesen Nutzen erläutern. Die niedrigste Varianzaufklärung konnte für das Merkmal Interesse an Wissenschaft festgestellt werden. Hier wäre es vermutlich wichtig, dass Studierende Forschungsbefunde und Theorie kennenlernen, durch die bei ihnen ein konkreter Nutzen deutlich wird und sie eine Hilfe für ihr schulisches Handeln feststellen. In zukünftigen Studien sollte zudem stärker erfasst werden, wie die weiteren Anforderungen des Praktikums mit denen des Forschenden Lernens zusammenwirken, da davon ausgegangen wird, dass sich gegenseitige Beeinflussungen ergeben. Insgesamt zeigt sich, dass die erwarteten und in anderen Studien berichteten Herausforderungen bei der Gestaltung Forschenden Lernens auch am hiesigen Standort bestehen. Die von Studierenden oft gesehene mangelnde Relevanz Forschenden Lernens (Göbel et al., 2016; Streblow \& Barsch, 2017) kann jedoch durch eine klare Transparenz der Ziele sowie des Nutzens und die damit verbundene Reflexion des Zusammenhangs zwischen Forschung und Schulpraxis erhöht werden. Gleichzeitig ist ein Bezug zur Praktikumsschule alleine nicht ausreichend, um bei Studierenden die wahrgenommene Forschungskompetenz zu erhöhen. Hierzu ist außerdem eine professionelle Unterstützung im Forschungsprozess (Fichten, 2017) von Bedeutung. Für die Stärkung der Möglichkeiten der Studierenden, reflexiv Theorie und Praxis aufeinander zu beziehen, ist der Bezug zum Praktikum hingegen von Bedeutung (Korthagen, 2010).

Die Studie leistet einen Beitrag zum Verständnis von Prozessen des Forschenden Ler- nens im Kontext des Praxissemesters in der Lehrkräftebildung, für welches zwar Forschendes Lernen theoretisch und politisch gefordert wird, empirisch jedoch wenig untersucht ist. Die Ergebnisse dieser Studie ordnen sich insgesamt erwartungsgerecht in bisherige Ergebnisse ein. Durch die Vernetzung der drei Bedingungsfaktoren Forschenden Lernens wird deutlich, dass zwar ein Praxissemester eine Gelegenheit bietet, für Studierende sinnhaft Forschendes Lernen zu betreiben, dies jedoch erst durch eine professionelle, für Studierende in den Zielen transparente und schulbezogene Unterstützung gelingt.

\section{Grenzen der vorliegenden Studie}

Durch das Design und die Datenstruktur der vorliegenden Studie ergeben sich mehrere Limitationen. So können weder Effekte der Gruppenzusammensetzungen in den jeweiligen Seminaren zum Forschenden Lernen noch Bedingungen der jeweiligen Praktikumsschule in die Untersuchung einbezogen werden. Weiterhin basieren auch die Aussagen zum Kompetenzgewinn auf Selbsteinschätzungen der Studierenden, sodass hier nicht der tatsächliche Kompetenzgewinn abgebildet werden kann. Durch die teilweise geringe Anzahl an Items pro Skala ist zudem davon auszugehen, dass nicht alle Konstrukte in ihrer ganzen Breite erfasst werden konnten. Das querschnittliche Erhebungsdesign, welches in diesem Fall gewählt wurde, um eine hohe Beteiligung der Studierenden zu erreichen, lässt keine Abbildung direkter Wirkungszusammenhänge zu, sodass die hier dargestellten Zusammenhänge durch weiterführende, experimentelle bzw. Pre-Post-Designs geprüft werden müssten. Gleichwohl können die hier dargestellten Ergebnisse Hinweise für die Gestaltung solcher Studien liefern. Für das langfristige Ziel einer forschenden Lebrkraft (Klewin \& Koch, 2017) bleibt zudem unklar, wie die hier dargestellten Zieldimensionen mit dem tatsächlichen Verhalten als Lehrkraft in der Schule in Verbindung stehen, was längsschnittliche Designs erforderlich machen würde. 


\section{Implikationen}

Für die weitere Forschung ergeben sich daraus Fragen, mit welchen konkreten Elementen der Zusammenarbeit aus Schule und Universität Forschendes Lernen im Kontext von Langzeitpraktika unterstützt werden kann, sodass es für Studierende zum Aufbau von Forschungskompetenz und einer kritisch-reflexiven Grundhaltung beiträgt und gleichzeitig Studierende ein Interesse an Forschung als Lehrkraft entwickeln. Die hier dargestellten Ergebnisse können für weitere, insbesondere auch längsschnittliche, experimentelle oder im Pre-PostDesign angelegte Studien Anregungen geben. Für die Praxis der Lehrkräftebildung konnte in dieser Studie gezeigt werden, dass Studierende bei Lehrveranstaltungen zum Forschenden Lernen nicht immer einen direkten Gewinn für sich sehen, dies jedoch durch transparente Ziele, Bezüge zur Praktikumsschule und Unterstützung im Forschungsprozess verbessert werden kann. Entsprechend wird empfohlen, dass auf struktureller Ebene die Ziele des Forschenden Lernens noch intensiver zwischen Schulen und Lehrenden diskutiert werden und somit ein stärkerer Konsens hierzu zwischen Lehrenden und Schulen erreicht werden kann. Hierbei gilt es besonders in den Fokus zu nehmen, wie Forschendes Lernen im Kontext eines Langzeitpraktikums gelingen kann, sodass es nicht zu einer Überforderung der Studierenden kommt. Weiterhin wird empfohlen, das Thema verstärkt in Fortbildungsprogramme für universitäres Personal und schulische Mentorinnen und Mentoren und somit auf individueller Ebene wirksam aufzunehmen, da Studierende nur durch eine gute Begleitung zum Forschenden Lernen angeregt werden können. Am Standort bearbeiten wir diese Herausforderungen in Forschung und Lehre aktuell und zukünftig weiter.

\section{Literatur}

BAK [Bundesassistentenkonferenz]. (1970). Forschendes Lernen - Wissenschaftliches Prüfen (Schriften der Bundesassistentenkonferenz Nr. 5). Bonn.

Büsing, A., Gehrs, V., Mochalski, A., Nakamura, Y. \& Treichel, B. (2017). Profile Forschenden Lernens - Das
Osnabrücker Konzept als Beispiel aus Niedersachsen. In R. Schüssler, A. Schöning, V. Schwier, S. Schicht, J. Gold \& U. Weyland (Hrsg.), Forschendes Lernen im Praxissemester: Zugänge, Konzepte, Erfahrungen (S. 111 118). Bad Heilbrunn: Julius Klinkhardt.

Feindt, A. (2006). Studentische Forschung im Lehramtsstudium: Eine fallrekonstruktive Untersuchung studienbiografischer Verläufe und studentischer Forschungspraxen. Unveröffentlichte Dissertation, Universität Oldenburg, Oldenburg. https://dx.doi.org/10.2307/j.ctvdf0 193

Fichten, W. (2017). Forschendes Lernen in der Lehrerbildung. In R. Schüssler, A. Schöning, V. Schwier, S. Schicht, J. Gold \& U. Weyland (Hrsg.), Forschendes Lernen im Praxissemester: Zugänge, Konzepte, Erfahrungen (S. 30-38). Bad Heilbrunn: Julius Klinkhardt.

Fichten, W. \& Meyer, H. (2014). Skizze einer Theorie forschenden Lernens in der Lehrer innenbildung. In E. Feyerer (Hrsg.), Beiträge zur Bildungsforschung: Nr. 1 . Last oder Lust? Forschung und Lehrer_innenbildung (S. 11-42). Münster: Waxmann.

Gess, C., Deicke, W. \& Wesels, I. (2017). Kompetenzentwicklung durch Forschendes Lernen. In H. A. Mieg \& J. Lehmann (Hrsg.), Forschendes Lernen: Wie die Lehre in Universität und Fachhochschule erneuert werden kann (S. 79-90). Frankfurt a. M.: Campus Verlag.

Göbel, K., Ebert, A. \& Stammen, K.-H. (2016). Ergebnisse der ersten Evaluation des Praxissemesters in Nordrhein-Westfalen. In Ministerium für Schule und Weiterbildung (Hrsg.), Schule NRW. Das Praxissemester auf dem Prüfstand. Zur Evaluation des Praxissemesters in Nordrhein-Westfalen (Beilage November 2016). Düsseldorf.

Healey, M. (2005). Linking research and teaching exploring disciplinary spaces and the role of inquiry-based learning. In R. Barnett (Ed.), Reshaping the university: New relationships between research, scholarship and teaching (pp. 67-78). Berkshire: Open University Press.

Helsper, W. \& Kolbe, F.-U. (2002). Bachelor/Master in der Lehrerbildung. Potential für Innovation oder ihre Verhinderung? Zeitschrift für Erziehungswissenschaft, 5, 384-400. https://dx.doi.org/10.1007/s11618-002$0057-\mathrm{z}$

Hofer, R. (2013). Forschendes Lernen in der Lehrerinnenund Lehrerbildung: Widersprüchliche Anforderungen zwischen Forschung und Profession. Beiträge zur Lehrerinnen- und Lehrerbildung 31, 310-320.

Homt, M. \& Ophuysen, S. van. (2018). Gelingensbedingungen für den Aufbau einer forschenden Grundhaltung im Praxissemester - eine qualitative vergleichende Fallstudie. In L. Pilypaitytė \& H.-S. Siller (Hrsg.), Schulpraktische Lehrerprofessionalisierung als Ort der Zusammenarbeit (S. 255-260). Wiesbaden: Springer. https://dx.doi.org/10.1007/978-3-658-170 86-8_19

Huber, L. (2009). Warum Forschendes Lernen nötig und möglich ist. In L. Huber, J. Hellmer \& F. Schneider (Hrsg.), Forschendes Lernen im Studium (S. 9-35). Bielefeld: Universitätsverlag Webler.

Huet, I. (2017). Research-based education as a model to change the teaching and learning environment in STEM disciplines. European Journal of Engineering Education, 43, 725-740. https://dx.doi.org/10.1080/030 43797.2017.1415299 
Klewin, G. \& Koch, B. (2017). Forschendes Lernen ohne forschende Lehrkräfte? Die Deutsche Schule, 109, $58-$ 69.

Koch-Priewe, B. \& Thiele, J. (2009). Versuch einer Systematisierung der hochschuldidaktischen Konzepte zum Forschenden Lernen. In B. Roters, R. Schneider, B. Koch-Priewe, J. Thiele \& J. Wildt (Hrsg.), Forschendes Lernen im Lehramtsstudium: Hochschuldidaktik, Profes sionalisierung, Kompetenzentwicklung (S. 271-292). Bad Heilbrunn: Julius Klinkhardt.

Köker, A. \& Störländer, C. (2017). Studentische Forschung im Praxissemester. In T. Burger \& N. Miceli (Hrsg.), Lehrbuch. Empirische Forschung im Kontext Schule: Ein führung in theoretische Aspekte und methodische Zugänge (S. 359-371). Wiesbaden: Springer. https://dx.doi. org/10.1007/978-3-658-15437-0_23

Korthagen, F. A. (2010). How teacher education can make a difference. Journal of Education for Teaching, 36 407-423. https://dx.doi.org/10.1080/02607476.201 0.513854

Lehmann, J. \& Mieg, H. A. (Hrsg.). (2018). Forschendes Lernen: Ein Praxisbuch. Potsdam: Verlag der Fachhochschule Potsdam.

Muthén, L. K. \& Muthén, B. O. (2017). Mplus User Guide. Los Angeles, CA: Muthén \& Muthén.

Niedersächsisches Ministerium für Wissenschaft und Kultur. (2014). Vereinbarung zur Implementierung einer Praxisphase in die viersemestrigen Masterstudiengänge für das Lehramt an Grundschulen und das Lehramt an Haupt- und Realschulen. Zugriff am 2.4.2019 unter http://www.mwk.niedersachsen.de/download/91274/ Kooperationsvereinbarung_vom_21.10.2014.pdf

Obolenski, A. \& Meyer, H. (Hrsg.). (2006). Forschendes Lernen: Theorie und Praxis einer professionellen LehrerIn nenausbildung (2., aktualisierte Aufl.). Oldenburg: Didaktisches Zentrum Univ.

Ohm, U. \& Zörner, A. (2019). Gelegenheiten Forschenden Lernens im Fachgebiet Deutsch als Zweitsprache im Praxissemester. Theoretische Modellierung und empirische Analysen auf der Basis narrativer Interviews. Herausforderung Lehrer_innenbildung, 2(2), 124-154. https://dx.doi.org/10.4119/UNIBI/hlz-134

Piaget, J. (1976). Piaget's Theory. In B. Inhelder, H. H. Chipman \& C. Zwingmann (Eds.), Piaget and his school (pp. 11-23). Berlin: Springer. https://dx.doi.org/10. 1007/978-3-642-46323-5_2

Rothland, M. \& Boecker, S. K. (2014). Wider das Imitationslernen in verlängerten Praxisphasen: Potenzial und Bedingungen des Forschenden Lernens im Praxis semester. Die Deutsche Schule, 106, 386-397.

Riewerts, K., Weiß, P., Wimmelmann, S., Saunders, C. Beyerlin, S., Gotzen, S., ... Gess, C. (2018). Forschendes Lernen entdecken, entwickeln, erforschen und evaluieren. Die Hochschullehre, 4, 389-406.

Schermelleh-Engel, K., Moosbrugger, H. \& Müller, H. (2003). Evaluating the Fit of Structural Equation Models: Tests of significance and descriptive goodness-offit measures. Methods of Psychological Research Online, $8(2), 23-74$

Schön, D.A. (1983). The reflective practitioner: How professionals think in action. New York, NY: Basic Books.

Schüssler, R. \& Schöning, A. (2017). Forschendes Lernen im Praxissemester - Potential und Ausgestaltungsmöglichkeiten. In R. Schüssler, A. Schöning, V. Schwier, S.
Schicht, J. Gold \& U. Weyland (Hrsg.), Forschendes Lernen im Praxissemester: Zugänge, Konzepte, Erfahrungen (S. 39-50). Bad Heilbrunn: Julius Klinkhardt.

Schüssler, R., Schöning, A., Schwier, V., Schicht, S., Gold, J. \& Weyland, U. (Hrsg.). (2017). Forschendes Lernen im Praxissemester: Zugänge, Konzepte, Erfahrungen. Bad Heilbrunn: Julius Klinkhardt.

Spies, A., Michaelis, J., Gerhein, U. \& Hinsch, V. (2017). Forschendes Lernen in Niedersachsen - Das Oldenburger Praxissemester. In R. Schüssler, A. Schöning, V. Schwier, S. Schicht, J. Gold \& U. Weyland (Hrsg.), Forschendes Lernen im Praxissemester: Zugänge, Konzepte, Erfahrungen (S. 104-110). Bad Heilbrunn: Julius Klinkhardt.

Streblow, L. \& Barsch, S. (2017). Evaluation im Praxissemester. In R. Schüssler, A. Schöning, V. Schwier, S Schicht, J. Gold \& U. Weyland (Hrsg.), Forschendes Lernen im Praxissemester: Zugänge, Konzepte, Erfah rungen (S. 70-80). Bad Heilbrunn: Julius Klinkhardt.

Terhart, E. (Hrsg.). (2000). Perspektiven der Lehrerbildung in Deutschland: Abschlussbericht der von der Kultusministerkonferenz eingesetzten Kommission. Beltz Pädagogik. Weinheim: Beltz.

Weyland, U. (2012). Expertise zu den Praxisphasen in der Lehrerbildung in den Bundesländern. Hamburg: Landesinstitut für Lehrerbildung und Schulentwicklung. Zugriff am 2.4.2019 unter http://li.hamburg.de/con tentblob/3305538/data/pdf-studie-praxisphasen-inder-lehrerbildung.pdf

Weyland, U. \& Wittmann, E. (2015). Langzeitpraktika in der Lehrerausbildung in Deutschland: Stand und Perspektiven. Journal für LehrerInnenbildung, 15(1), 8-21.

Weyland, U. \& Wittmann, E. (2017). Praxissemester en vogue. In R. Schüssler, A. Schöning, V. Schwier, S Schicht, J. Gold \& U. Weyland (Hrsg.), Forschendes Lernen im Praxissemester: Zugänge, Konzepte, Erfahrungen (S. 17-29). Bad Heilbrunn: Julius Klinkhardt.

Wissenschaftsrat. (2001). Empfehlungen zur zukünftigen Struktur der Lehrerausbildung. Berlin.

Wissenschaftsrat. (2006). Empfehlungen zur zukünftigen Rolle der Universitäten im Wissenschaftssystem. Berlin.

Zimbardi, K. \& Myatt, P. (2012). Embedding undergraduate research experiences within the curriculum: A cross-disciplinary study of the key characteristics guiding implementation. Studies in Higher Education, 39, 233-250. https://dx.doi.org/10.1080/03075079.201 1.651448

\section{Timo Beckmann}

Leuphana Universität Lüneburg

Fakultät Bildung

Universitätsallee 1

D-21335 Lüneburg

E-Mail: timo.beckmann@uni.leuphana.de

\section{Prof. Dr. Timo Ehmke}

Leuphana Universität Lüneburg

Institut für Bildungswissenschaft

Universitätsallee 1

D-21335 Lüneburg

E-Mail: timo.ehmke@uni.leuphana.de 